Unfallchirurg 2009 $112: 533$

DOI 10.1007/s00113-009-1628-8

Online publiziert: 30. April 2009

๑) Springer Medizin Verlag 2009

Redaktion

H. Siebert, Schwäbisch Hall

\author{
S. Ruchholtz \\ Klinik für Unfall-, Hand- und Wiederherstellungschirurgie, \\ Universitätsklinikum Giessen/Marburg, Standort Marburg
}

\title{
Kostenkontrolle in der Schwerverletztenversorgung durch das TraumaNetzwerk DGU
}

zelfällen extrem stark variiert, zu einem Fallwert dar.

Als wesentliche variable Parameter, die zur Berechnung einzelner Fallwerte herangezogen werden sollten, werden die Operationsdauer, Aufenthaltsdauer auf der Intensivstation und Verletzungsschwere als relevante kostenabhängige $\mathrm{Pa}$ rameter aufgeführt.

Neben den Parametern zur verbesserten Abschätzung des tatsächlichen Aufwands zeigen die Autoren auf, dass insbesondere bei der Entwicklung des TraumaNetzwerks DGU großen Chancen für eine gerechtere Berechnung und eine Minimierung der Kosten bei der Versorgung von schwerverletzten Patienten bestehen.

Ein entscheidender Aspekt des TraumaNetzwerks DGU, das sich mittlerweile aus 59 regionalen Traumanetzwerken mit mehr als 700 teilnehmenden Kliniken deutschlandweit zusammensetzt, ist die gemeinsame standardisierte Erfassung der Verläufe schwerverletzter Patienten auf Basis des TraumaRegisters. Über das Kostenschätzungsmodul des Traumaregisters können dann erstmalig deutschlandweit zuverlässige Daten zu Behandlungskosten in allen Kliniken der 3 unterschiedlichen Kategorien eines Traumanetzwerks aufgezeigt werden.

Es ist zu erwarten, dass durch die Konzentrierung der besonders aufwendigen Fälle in regionalen und überregionalen Traumazentren die hohen Vorhaltungskosten zur Schwerverletztenversorgung optimaler genutzt werden können.

Eine weitere Kostenersparnis ist v. a. für solche Fälle zu erwarten, bei denen durch eine verbesserte prä- und frühklinische Versorgung die Aufenthaltsdauer auf Intensivstationen sowie die Operationsdauer insbesondere unter Berücksichtigung möglicher Revisionen bei Komplikationen vermindert werden können.

Wesentliche Voraussetzung ist jedoch weiterhin die Fortführung des seit Beginn bestehenden konstruktiven Dialogs zwischen dem InEK und Vertretern der Deutschen Gesellschaft für Unfallchirurgie, um dem kontinuierlichem Problem einer deutlichen Unterdeckung der Behandlungsvergütung entgegen zu wirken.

Zielführend erscheint es aus Sicht des Kommentators, dabei zukünftig auch im Bereich der Kostenkalkulation von dem Begriff „Polytrauma“ Abstand zu nehmen, denn sowohl der finanzielle als auch klinische Aufwand sind im Wesentlichen von der Verletzungsschwere ggf. auch einzelner Organsysteme (z. B. schweres Schädel-Hirn-Trauma oder schwere beidseitige Thoraxkontusion) bestimmt und nicht allein durch die Tatsache, das 3 Körperregionen Verletzungen aufweisen. Die von den Autoren herausgearbeiteten Parameter könnten neben der Verletzungsschwere den tatsächlichen Behandlungs- und Kostenaufwand verbessert wiedergeben.

\section{Korrespondenzadresse \\ Prof. Dr. S. Ruchholtz}

Klinik für Unfall-, Hand- und

Wiederherstellungschirurgie,

Universitätsklinikum Giessen - Marburg;

Standort Marburg

Baldingerstraße, 35033 Marburg

ruchholt@med.uni-marburg.de

Interessenkonflikt. Der korrespondierende Autor

gibt an, dass kein Interessenkonflikt besteht. tung stellt weiterhin die individuelle $\mathrm{Zu}$ ordnung der Behandlung, die in den Ein- 